\title{
A Core Outcome Set for Pediatric Critical Care
}

Ericka L. Fink, MD, MS ${ }^{1}$; Aline B. Maddux, MD, MSCS²; Neethi Pinto, MD, MS³ Samuel Sorenson, $\mathrm{BS}^{4}$; Daniel Notterman, $\mathrm{MD}^{5}$; J. Michael Dean, $\mathrm{MD}^{4}$; Joseph A Carcillo, MD; Robert A Berg, MD; Athena Zuppa, MD³; Murray M Pollack, MD; Kathleen L Meert, MD'; Mark W Hall, MD; Anil Sapru, MDㅜ; Patrick S McQuillen, MD ${ }^{10}$; Peter M Mourani, MD²; David Wessel, MD ${ }^{6}$; Deborah Amey ${ }^{11}$; Andrew Argent, MD ${ }^{12}$; Werther Brunow de Carvalho, MD, PhD ${ }^{13}$; Warwick Butt, FRACP, $\mathrm{FCICM}^{14}$; Karen Choong, MB, BCh, MSc ${ }^{15}$; Martha A.Q. Curley, RN, PhD'27; Maria del Pilar Arias Lopez, MD ${ }^{16}$; Demet Demirkol, MD ${ }^{17}$; Ruth Grosskreuz, MD, CCRP²; Amy J. Houtrow, MD, PhD, MPH ${ }^{18}$; Hennie Knoester, MD, PhD ${ }^{19}$; Jan Hau Lee, MBBS, MRCPCH, MCl20; Debbie Long, RN, $\mathrm{PhD}^{21}$; Joseph C. Manning, PhD, RN22; Brenda Morrow, PhD, PT ${ }^{23}$; Jhuma Sankar, MD24; Beth S. Slomine, $\mathrm{PhD}^{25}$; McKenna Smith, $\mathrm{BS}^{4}$; Lenora M. Olson, $\mathrm{PhD}^{4}$; R. Scott Watson, MD, MPH ${ }^{26}$; the POST-PICU Investigators of the Pediatric Acute Lung Injury and Sepsis Investigators (PALISI) Network and the Eunice Kennedy Shriver National Institute of Child Health and Human Development Collaborative Pediatric Critical Care Research Network (CPCCRN)

\section{Author Contact Info}

1 - Department of Critical Care Medicine, UPMC Children's Hospital of Pittsburgh, Pittsburgh, PA, USA 
2 - Department of Pediatrics, Critical Care Medicine, University of Colorado School of Medicine, Children's Hospital Colorado, Aurora, CO, USA

3 - Department of Anesthesiology and Critical Care Medicine, Children's Hospital of Philadelphia, Philadelphia, PA, USA

4 - Department of Pediatrics, University of Utah School of Medicine, Salt Lake City, UT, USA

5 - Department of Molecular Biology, Princeton University, Princeton, NJ, USA

6 - Department of Pediatrics, Children's National Hospital, Washington, DC, USA

7 - Department of Pediatrics, Children's Hospital of Michigan, Detroit, MI, USA

8 - Department of Pediatrics, Nationwide Children's Hospital, Columbus, OH, USA

9 - Department of Pediatrics, Mattel Children's Hospital, University of California Los Angeles, Los Angeles, CA, USA

10 - Department of Pediatrics, Benioff Children's Hospital, University of California, San Francisco, San Francisco, CA, USA 
11 - Advocate, Collaborative Pediatric Critical Care Research Network Family

Collaborative, Great Falls, Virginia, USA

12 - Department of Paediatrics and Child Health, University of Cape Town, and Red Cross War Memorial Children's Hospital, Cape Town, South Africa

13 - Department of Pediatrics, University of São Paulo, Brazil

14 - Intensive Care Department of Paediatrics, The Royal Childrens Hospital, Melbourne, Australia

15 - Departments of Pediatrics and Critical Care, McMaster University, Ontario, Canada

16 - Department of Pediatric Critical Care, Hospital de Niños Dr R. Gutierrez, Buenos Aires, Argentina

17 - Istanbul University, Child Health Institute and Istanbul Faculty of Medicine, Department of Pediatric Intensive Care, Istanbul, Turkey

18 - Department of Physical Medicine \& Rehabilitation, University of Pittsburgh, Pittsburgh, PA, USA

19 - Department of Paediatrics, Centrum Universiteit van Amsterdam, the Netherlands 
20 - Department of Pediatric Subspecialities, KK Women's and Children's Hospital, Singapore

21 - Paediatric Intensive Care Unit, Queensland Children's Hospital, and PCCRG, Centre for Children's Health Research, The University of Queensland, Australia

22 - Children and Young People Health Research, School of Health Sciences, University of Nottingham and Nottingham Children's Hospital, Nottingham University Hospitals NHS Trust, United Kingdom

23 - Department of Paediatrics and Child Health, University of Cape Town, South Africa

24 - Department of Pediatrics, All India Institute of Medical Sciences, Chandigarh, India

25 - Department of Neuropsychology, Kennedy Krieger Institute and Department of Psychiatry and Behavioral Sciences, Johns Hopkins University School of Medicine, Baltimore, MD, USA

26 - Department of Pediatrics, University of Washington School of Medicine and Center for Child Health, Behavior, and Development, Seattle Children's Research Institute, Seattle, WA, USA 
27 - Department of Family and Community Health (Nursing), Anesthesiology and Critical Care (Perelman School of Medicine), University of Pennsylvania; Research Institute, Children's Hospital of Philadelphia, Philadelphia, PA, USA

\section{Corresponding Author:}

Ericka L. Fink, MD, MS

Division of Pediatric Critical Care Medicine

UPMC Children's Hospital of Pittsburgh

4401 Penn Avenue

Faculty Pavilion, 2nd floor

Pittsburgh, PA 15224

Phone: 412-692-5164

Fax: 412-692-6076

Email: finkel@ccm.upmc.edu

\section{Author contributions}

ELF, RSW, LMO, NP, and ABM: Substantial contributions to study conception/design, acquisition, analysis, and interpretation of data, and manuscript preparation. SS provided management for this work. DN, JMD, JAC, RAB, AZ, MMP, KLM, MWH, AS, PSM, PMM contributed to design, manuscript revision. AA, BS, BM, DA, DL, DD, HK, JHL, JS, JCM, KC, MPAL, MAQC, RG, WB, WBC contributed to design, manuscript revision. All authors are accountable for all aspects of the work in ensuring that 
questions related to the accuracy or integrity of any part of the work are appropriately investigated and resolved and approved the final version for submission.

\section{Conflicts of Interest and Source of Funding:}

Drs. Fink, Maddux, Notterman, Dean, Carcillo, Berg, Zuppa, Pollack, Meert, Hall, Sapru, McQuillen, Mourani, Maddux, Watson, and Wessel and Mr. Sorenson have grant support from the National Institutes of Health.

Dr. Manning receives support from the National Institute for Health Research.

The remaining authors report no conflicts.

Funding for this project was provided by the Eunice Kennedy Shriver National Institute of Child Health and Human Development Collaborative Pediatric Critical Care Research Network (CPCCRN). It was approved by the CPCCRN Steering Committee and funded by grant numbers U01-HD049934, UG1-HD083171, UG1-HD050096, UG1-HD063108, UG1-HD049983, UG1-HD049981, UG1-HD083170, and UG-HD083166. Additional support from NICHD K23HD096018 (Maddux) and the Francis Family Foundation (Maddux).

Key Words: Child; Critical Care; Outcome Assessment; Post Intensive Care Syndrome; Family

Total word count: 2722 


\section{ABSTRACT}

\section{Objective}

More children are surviving critical illness but are at risk of residual or new health conditions. An evidence-informed and stakeholder-recommended core outcome set is lacking for pediatric critical care outcomes. Our objective was to create a multinational, multi-stakeholder-recommended Pediatric Critical Care Core Outcome Set for inclusion in clinical and research programs.

\section{Design}

A 2-round modified Delphi electronic survey was conducted with 333 invited research, clinical, and family/advocate stakeholders. Stakeholders completing the first round were invited to participate in the second. Outcomes scoring > 69\% "critical" and $<15 \%$ "not important" advanced to round 2 with write-in outcomes considered. The Steering Committee held a virtual consensus conference to determine the final components.

\section{Setting}

Multinational survey.

\section{Patients}

Stakeholder participants from 6 continents representing clinicians, researchers, and family/advocates.

Main Results 
Overall response rates were $75 \%$ and $82 \%$ for each round. Participants voted on 7 Global Domains and 45 Specific Outcomes in Round 1, and 6 Global Domains and 30 Specific Outcomes in Round 2. Using Overall (3 stakeholder groups combined) results, consensus was defined as outcomes scoring > 90\% "critical" and $<15 \%$ "not important" and were included in the final PICU COS: 4 Global domains (Cognitive, Emotional, Physical and Overall Health) and 4 Specific outcomes (Child Health-Related Quality of Life, Pain, Survival, and Communication). Families $(n=21)$ suggested additional critically important outcomes that did not meet consensus, which were included in the PICU COS - Extended.

\section{Conclusions}

The PICU Core Outcome Set and PICU COS-Extended are multi-stakeholderrecommended resources for clinical and research programs that seek to improve outcomes for children with critical illness and their families.

Words in abstract: 264 


\section{Introduction}

Approximately 480,000 children and young adults $<20$ years old are admitted to pediatric intensive care units (PICUs) annually at a cost of $\$ 8$ billion in the United States alone ${ }^{1,2}$. Mortality has decreased to $2-4 \%$ in high-resource settings ${ }^{3}$. However, child and family survivorship and recovery are frequently affected by ongoing and/or new impairments in physical, emotional, cognitive, and/or social health functioning, termed Post-Intensive Care Syndrome-pediatrics ${ }^{4}$.

The vast majority of pediatric critical care research studies employ short-term (inhospital) physiologic or mortality outcomes, with few studies assessing outcomes posthospital discharge ${ }^{5-9}$. Researchers report that key monetary and resource barriers to carrying out high quality trials include assessment of outcomes post-hospital discharge

10. Furthermore, heterogeneity in outcome measures and time points selected in studies prohibits systematic review and meta-analysis ${ }^{11}$.

The status quo for PICU outcomes is shifting towards inclusion of outcomes prioritized by providers, patients, and families ${ }^{12-14}$ rather than solely by investigators. Core Outcome Sets (COS), defined as "a patient outcome, health-related condition, or aspects of health that relevant stakeholders agree are essential to assess in all clinical research studies evaluating outcomes", have been developed and implemented successfully for other critically ill populations, but not for pediatric critical care ${ }^{15,16}$. Additionally, use of a COS allows for increased ability to compare outcomes across studies and populations and decreases the potential for reporting bias ${ }^{17}$.

Our objective was to develop a multi-stakeholder-informed PICU-COS. The product of this effort is a minimum set of outcome domains that should be incorporated 
into clinical and research programs to evaluate outcomes of critically ill children and families. 


\section{Materials and Methods}

\section{Study Design}

We incorporated recommendations for methodology and quality standards for design and reporting for $\operatorname{COS}^{18-20}$. This manuscript reports on the Delphi consensus process yielding the final PICU COS. Additional description of the study protocol ${ }^{21}$ and preliminary aims were published ${ }^{6,22,23}$. This project was approved by the University of Utah Institutional Review Board.

\section{Modified, international Delphi consensus - process overview}

Delphi content. Investigators created a list of unique Global Domains and Specific Outcomes from a scoping review, qualitative study, and other relevant sources 13,14,16,24. Domains and Outcomes were paired with lay definitions that were reviewed by the Collaborative Pediatric Critical Care Research Network (CPCCRN)'s Family Network Collaborative, composed of 1-2 family volunteers from each of the 7 centers. The Steering Committee approved the Domains, Outcomes, and lay definitions. The CPCCRN's Data Coordinating Center prepared the Delphi software.

Stakeholders and Steering Committee members. The Steering Committee was recruited by the primary investigators. Candidates were invited with consideration for expertise in PICU Outcomes and diversity in background, region, and gender. All were fluent in English.

The Steering Committee invited 333 participants from three stakeholder groups Research, Clinical, and Family - via an introductory email. After consenting, stakeholders were asked to respond based on their own perspective, except for those 
who represented an organization. Members of the Steering Committee were included as stakeholders as they represented leaders in pediatric critical care outcomes and families of children with critical illness.

Modified Delphi Consensus Methods. We planned for a minimum of two Delphi rounds to reach consensus. Panel members scored components using the Grading of Recommendations Assessment, Development and Evaluation (GRADE) Scale, which consists of a 9-point scale: "not important for inclusion" (scores 1-3), "important but not critical for inclusion" (scores 4-6), "critical for inclusion" (scores 7-9), and "unable to score" (score 10) ${ }^{25}$. Global Domains and their related Specific Outcomes were randomized into four different orders and randomly assigned to panel members each round. Stakeholders were given approximately 3 weeks to complete each round. Nonrespondents received a weekly personalized email, telephone call, or text reminder. Stakeholder response rates were calculated as the number of respondents who completed each round as a proportion of those for whom an email invitation was sent. Those who participated in Round 1 were invited for Round 2.

In Round 1, we recorded demographic information and assigned each consented participant a unique identifier. Respondents could propose novel outcomes. A priori criteria for an outcome domain from Round 1 to be included in Round 2 required $>70 \%$ of responses rating $\geq 7$ AND $<15 \%$ of response rating $<3$. The Steering Committee used consensus to confirm the outcomes panel for Round 2. The Steering Committee reviewed new outcome suggestions from Round 1 to ensure they represented a new contribution for inclusion in Round 2 
During Round 2 voting, respondents were provided aggregate responses from the first round for all stakeholders and by stakeholder group, their own response from Round 1, and new outcomes from Round 1. The Steering Committee used consensus to confirm the final COS components taking into account number of domains and importance based on scores by stakeholder group, ultimately as those in Round 2 with $>90 \%$ of responses rating $\geq 7$ AND $<15 \%$ of response rating $<3$.

Analysis and reporting. Each outcome's score was analyzed based on the total number of respondents who answered the question. We report measures of central tendency, score distribution, and score changes by round of PICU COS Global Domains and Specific Outcomes as well as those considered for inclusion. 


\section{Results}

\section{Steering committee and stakeholders}

The Steering Committee consisted of 23 members, including at least two representatives from each of the six continents and a heterogeneous group of clinical and research experts as well as a member from the family stakeholder group (Supplementary Table 1). The Steering Committee recommended a geographically diverse group of participants for each of the-stakeholder group members ( $n=333$ total): er groups $(n=333):$ Research $(n=59)$, Clinical $(n=226)$, and Family $(n=48)$

(Supplementary Table 2). The largest group, clinicians, included nurses, allied health practitioners, palliative care providers, physicians from multiple disciplines (e.g., pediatric critical care, physical medicine and rehabilitation, complex care pediatrics, pediatric surgery, and pulmonology), and others involved in the care of PICU patients during and after hospitalization (e.g., healthcare system and payor administrators). Researchers included authors who had published studies on post-pediatric intensive eare outcomes, research coordinators, and funding agency representatives. Family stakeholders included parents, guardians, adults who survived pediatric critical illness, and members of PICU-related advocacy groups. Table 1 describes stakeholder characteristics for those who responded to both rounds. Notably, there were more female respondents for each stakeholder group. Most family respondents were located in North America (81.0\%). All stakeholders reported at least some post-secondary education.

\section{Delphi Round 1}


The response rate for Round 1 was 251/333 (75.0\%) overall and 180/226 $(80.0 \%)$ for clinicians, $38 / 59(63.3 \%)$ for researchers, and $33 / 48$ (68.8\%) for family stakeholders. The Round 1 survey included 7 Global Domains and 45 Specific Outcomes (Figure 1). No outcomes met criteria for "not important for inclusion" among any stakeholder group (Supplemental Table 3). There was good general agreement in Global Domain scores among the groups (Supplemental Table 4). Family stakeholder group scores were generally higher than other groups for Specific Outcomes, especially for domains related to family function. Six Global Domains (all except Health Care Utilization) and 22 Specific Outcomes met the a priori cutoff of $70 \%$ of responses rating $\geq 7$ AND $<15 \%$ of response rating $<3$ for inclusion in Round 2 . Within Global Domains, 4 of 6 Specific Outcomes from cognitive function, 7 of 10 overall health, 4 of 5 physical function, 2 of 5 emotional health, 3 of 9 family function, 2 of 6 health care utilization, and no social function met this threshold.

The Steering Committee elected to include in Round 2 an additional 4 Specific Outcomes that had an overall score from Round 1 approaching the inclusion threshold (69-69.9\%) and were strongly regarded as "critical" by the Family stakeholder group (Sleep, Parent/ Legal Guardian Quality of Life, Child Participation, Hospital/Intensive Care Unit Readmission).

Participants submitted 61 write-ins from Round 1, resulting in 5 new outcomes voted on during Round 2. These included the division of Parent/Legal Guardian Overall Health into 4 Specific Outcomes (Emotional, Physical, Social, and Overall Function) as 
well as the addition of a new outcome (New Medical Conditions or Diseases). The remaining write-in responses were either outcome instruments or not new, unique outcomes and were not included. Definitions for some outcomes were modified to clarify criteria (Supplemental Table 5).

\section{Delphi Round 2}

The Round 2 survey had a response rate of 206/251 (82.1\%): 150/180 (83.3\%) for clinicians, 35/38 (92.1\%) for researchers, and 21/33 (63.6\%) for family/advocacy stakeholder groups. The Round 2 survey included 6 Global Domains and 30 Specific Outcomes. No outcomes met criteria for "not important for inclusion" among any stakeholder group (Supplemental Table 6). Some participants made relatively minor changes to their scores between rounds (Figures $2 \underline{a}$ and $2 b$ ). The Steering Committee approved four Global Domains (Overall, Cognitive, Physical, and Emotional Function) in addition to 4 Specific Outcomes (Child Quality of Life, Survival, Pain, and Communication) that are ultimately included in the final COS (Table 2). Four of 7 Family Function Specific Outcomes met a priori criteria for inclusion, but none met the adjusted criteria

(Supplemental Table 7,

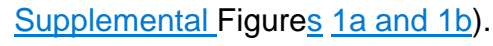

Further, hospital and ICU readmission were among the lowest-scoring outcomes in Further, hospital and ICU readmission were among the lowest-scoring outcomes in Round 2. The lowest scoring Specific Outcomes were some of the newly 
added family outcomes which had widely disparate scoring among Family, Clinician and Research stakeholders.

Scoring disparities among stakeholder groups were discussed by the Steering Committee via webinar. Ultimately, to recognize Family Stakeholder priorities, the Steering Committee recommended creation of a PICU COS - Extended tool

(Table 2, Supplemental Figures 1a and 1b).

The PICU COS - Extended includes 14 Specific Outcomes that met the "critical for inclusion" threshold by $>90 \%$ Family Stakeholders from the Global Domains Overall Health, and Family, Emotional, and Physical Function. 


\section{Discussion}

We developed a multinational, multi-stakeholder and evidence informed COS for clinical and research use in pediatric critical illness. Two rounds of a modified Delphi survey led to consensus. A supplemental COS - Extended was also created by the Steering Committee to recognize outcomes important to family members that did not meet consensus.

No guidelines exist for the follow-up of pediatric critical care patients as they do No guidelines exist for the follow-up of pediatric critical care patients as they do for No guidelines exist for the follow-up of pediatric critical care patients as they do for $\underline{N}$ guidelines exist for the

follow-up of pediatric critical care patients as they do for neonates and infants with congenital heart disease ${ }_{-}^{3,12,26}$ or adults surviving critical illness ${ }^{16}$. Furthermore, pediatric critical care research programs infrequently include postdischarge outcomes

$5,7,10$. Finally, the status quo for outcome assessment for clinical care and for research has not yet evolved to include input from stakeholders such as patients and families ${ }^{27,28}$.

Development of a multi-stakeholder-informed COS to guide clinicians and researchers in choosing post-hospital discharge outcomes in addition to other outcomes vital to clinical and research aims will increase program $\underline{\text { value and facilitate evidence robustness }}^{15,}$

29 . 
We followed international guidelines

for the development of COS including a mixed-methods approach to the generation of outcomes and a multinational and multi-stakeholder Steering Committee and Delphi respondent panel 6,16,20,22.

The final PICU COS features the Global Outcome Domains of Cognitive, Emotional, and Physical Function and Overall Health. In addition, Specific Outcomes under Cognitive Function (Child Communication), and Overall Health (Child Survival, Health-Related Quality of Life, and Pain) were also included. All three stakeholder groups scored these outcomes as critically important, with some differences in most highly valued outcomes by group, including Global Emotional Function and Communication by Families, and Survival by Researchers and Families. Lasting emotional health effects in children and families affected by pediatric critical illness can be substantial, requiring monitoring and treatment ${ }^{30}$. Survival and pain are frequently assessed within the hospital epoch but not post-hospital discharge despite reports of late pediatric deaths and ongoing pain symptoms reports in adults ${ }^{9,31,32}$. Health-related quality of life, a subjective outcome incorporating a proxy/patient's perception of the interplay of multiple outcome domains, was the highest rated Specific Outcome and is one of the more commonly reported post-discharge outcomes in pediatric critical illness 8,33. Cognitive function post-discharge has been overwhelmingly assessed using measures of intelligence, memory, attention, and/or executive function; reports on child communication function are lacking ${ }^{34}$.

Patient and family stakeholders, despite placing generally greater importance on outcomes compared to the other two stakeholder groups, clearly show value 
preferences for certain outcomes compared to clinicians and researchers. Family functioning is impacted by pediatric critical illness and can also influence the trajectory of recovery and long-term functional outcomes ${ }^{35,36}$. Further, the scope and depth of post-hospital discharge problems with sleep and physical function, and post-traumatic stress in both children and parents may go unrecognized by many stakeholders outside of the Family group. Hence, we recognized the need for the Extended outcome set to promote the outcomes valued by families. Additional goals for inclusion of these additional outcomes are to stimulate and support awareness, education, and research across the inpatient-outpatient spectrum of stakeholders. Post-PICU follow-up clinics are beginning to service this need but more systematic investment is needed 3738 . Implementation of a COS would be facilitated by recommendations for specific instruments for each outcome in a Core Outcome Measurement Set ${ }^{17,39}$. Thus, the next task for our Steering Committee is to recommend feasible (e.g., low cost, widely available, minimal administration time), reliable, age-appropriate and validated measurement instruments along with recommendations for timing of assessments posthospital discharge to evaluate outcomes. Some outcomes in the COS may not have validated instruments available that meet these criteria and there may be overlap in some of the content of outcomes in the COS. These recommendations will require frequent reassessment as new information and outcome measures become available.

Finally, the last aim of the PICU COS program is to strategize for broad dissemination and implementation of the PICU COS. In addition to creation of a Steering Committee and Delphi stakeholders with diverse membership, we registered this program on the Core Outcome Measures in Effectiveness Trials' Initiative (COMET) 
website (http://www.comet-initiative.org/Studies/Details/1131). Further, we will publish the PICU COS and PICU COS - Extended on the CPCCRN website

(https://www.cpccrn.org/network-projects/) and Pediatric Acute Lung Injury and Sepsis

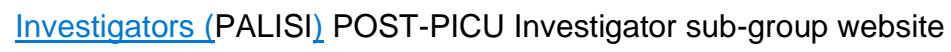
(https://www.palisi.org/subgroups, in development). We plan to submit abstracts and manuscripts for each program aim and secondary analyses to disseminate academically. We will provide approved fact sheets and infographics to all stakeholders for efficient dissemination to patient advocates and academic groups. We will develop an efficient process to monitor future use of the PICU COS in research proposals, grants, quality improvement initiatives, and publications. We will also lead a social media campaign to disseminate the COS after publication.

\section{Limitations}

Although we worked to recruit an equitable number of family stakeholders, this group had the smallest representation in the Delphi. Stakeholders had to be fluent to participate in the Delphi. Thus, we may have missed input from families and other

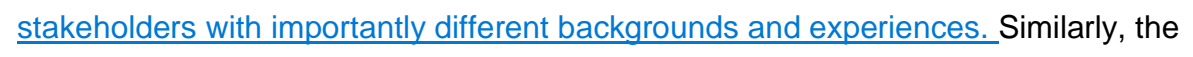
number and breadth of geographical distribution of representatives of research funding agencies, payors, and hospital administrators was relatively small; overall, stakeholders from North America were over-represented. The PICU COS was created to serve all children with critical illness. However, we recognize that children admitted to PICUs have a diverse range of ages; ethnicities; hospital admission condition, severity, and comorbidities; social determinants of health; family structures; geographical locations; 
quality of healthcare resources and access to care; each of which may require an additional personalized approach and evaluation of utility to outcomes selection.

We encourage re-evaluation of the PICU COS content every 5-10 years to improve upon methodology and evolution of best practices for post-discharge outcomes in this rapidly developing field. Finally, we recommend considering the inclusion of patients and families in other aspects of the clinical/research process in addition to outcomes choices. 


\section{Conclusions}

The PICU COS and PICU COS-Extended are multi-stakeholder-approved

resources for clinical and research programs that seek to more systematically study and improve outcomes for children with critical illness and their families. 
Acknowledgements. We acknowledge Dale Needham, MD, PhD (Johns Hopkins University, USA), Paula Williamson, PhD (University of Liverpool, UK), and Gabriel Frizzo Ramos, MD (University of São Paulo, Brazil), Tammara L. Jenkins, MSN, RN, PCNS-BC, FCCM (Program Officer, Pediatric Trauma and Critical Illness Branch) and Robert Tamburro, MD, MSc (Medical Officer, Pediatric Trauma and Critical Illness Branch) from the Eunice Kennedy Shriver National Institute of Child Health and Human Development; PALISI- POST-PICU Investigators (All USA unless specified): Samer Abu-Sultaneh, MD (Indiana University School of Medicine), Manzilat Akande, MD, MPH (The Children's Hospital of Oklahoma), Alicia M. Alcamo, MD, MPH (Children's Hospital of Philadelphia), Stefanie G. Ames, MD MS (David Geffen School of Medicine at University of California- Los Angeles), Reinis Balmaks, MD, PhD (Riga Stradins University), Anoopindar Bhalla, MD, MSCI (University of Southern California, Children's Hospital Los Angeles), Katherine Biagas, MD, FCCM, FAAP (Renaissance School of Medicine), Meredith Bone, MD, MSCI (Northwestern University Feinberg School of Medicine), LeeAnn M. Christie, MSN, RN (Dell Children's Medical Center), Karen Choong, MB, FRCP(C), MSc (McMaster University, Canada), Jonna Clark, MD, MA (University of Washington School of Medicine), Mike Cronin, MD, MPH (University of Washington School of Medicine), Sabrina Derrington, MD, MA, FAAP (Northwestern University Feinberg School of Medicine), Leslie A. Dervan, MD, MS (University of Washington School of Medicine), Jane (Lin) Di Gennaro, MD, MS (University of Washington School of Medicine), Idris Evans, MD, MSc (UPMC Children's Hospital of Pittsburgh), Reid WD Farris, MD, MS (University of Washington School of Medicine), Andy Geneslaw, MD (Columbia University Irving Medical Center), Denise M. Goodman 
MD MS, FCCM (Northwestern University Feinberg School of Medicine), Robert J.

Graham, MD (Boston Children's Hospital), Madhura Hallman, MD, MPH (University of Alabama at Birmingham), Mary E. Hartman, MD, MPH (Washington University in St. Louis), John C. Lin, MD (Washington University), Julia A. Heneghan, MD (University of Minnesota Masonic Children's Hospital), Jay M. Hunter, DNP, APRN, CPNP-AC, CCRN, CPN (University of California San Francisco), Kevin Hummel, MD (University of Utah), J. Dean Jarvis, BSN, MBA, RN, CCRP (Children's Hospital at DartmouthHitchcock), Jessica M. Jarvis, PhD (UPMC Children's Hospital of Pittsburgh); Elizabeth Y. Killien, MD, MPH (University of Washington School of Medicine), Sapna R.

Kudchadkar, MD, PhD (Johns Hopkins University), Mara Leimanis, PhD (Michigan State University), Simon Li, MD, MPH (New York Medical College), Laura Loftis (Baylor College of Medicine), Michele Loi, MD (University of Colorado), Debbie Long RN PhD (Queensland Children's Hospital), Peter M. Luckett, MD (University of Texas Southwestern Medical Center), Catherine Madurski, MD (Nemours Children's Health System), Maureen A. Madden MSN, RN, CPNP-AC, CCRN, FCCM (Rutgers Robert Wood Johnson Medical School), Joseph C. Manning, PhD, RN (Nottingham Children's Hospital), Mary McNally, BSRT, RRT (Children's Hospital at Dartmouth-Hitchcock), Julie Menzies, PhD, MSc, B(Nurs), RNC (Birmingham Children's Hospital), Kelly Michelson, MD, MPH, FCCM, FAAP (Northwestern University Feinberg), Lindy Moake, APRN, MSN, PCCNP (Children's Medical Center), Haifa Mtaweh, MD (University of Toronto, Canada), Sarah Murphy, MD (Harvard Medical School), Jennifer A Muszynski, MD, MPH (Nationwide Children's Hospital), Vinay Nadkarni, MD, MS (Children's Hospital of Philadelphia), Sholeen Nett, MD, PhD (Children's Hospital at Dartmouth- 
Hitchcock), Katie R. Nielsen, MD, MPH (University of Washington), AM Iqbal O'Meara, MD (Virginia Commonwealth University), Elizabeth Pace, MD (Case Western Reserve University), Andrew Prout, MD, MPH (University at Buffalo), Monique Radman, MD, MAS (University of Washington), Madiha Raees, MD (UPMC Children's Hospital of Pittsburgh), Brian Rissmiller, MD (Baylor College of Medicine), Joan S Roberts, MD (University of Washington), Shilpa Shah, DO (Children's Hospital Los Angeles), Steve L. Shein, MD (Case Western Reserve University), Marcy Singleton, ARNP (DartmouthHitchcock Medical Center), Katherine N. Slain, DO (Case Western Reserve University), Kristen Smith, MD, MS (University of Michigan), Alexis Topjian, MD, MSCE (Children's Hospital of Philadelphia), Chani Traube, MD (Weill Cornell Medical Center), Danielle Van Damme, DNP, CPNP-AC (University of Louisville), Sara K VandenBranden, MD (Rush University Medical Center), Ben White, MD, MA (University of Utah), Jane E. Whitney, MD MSCE (Harvard Medical School), Alan G. Woodruff, MD (Brenner Children's Hospital), Mekela Whyte-Nesfield, MD (Children's National Hospital), Lauren Yagiela, MD, MS (Children's Hospital of Michigan), and Jerry Zimmerman, MD, PhD, FCCM (University of Washington); and CPCCRN members Andrew Yates, MD (Nationwide Children's Hospital), John Berger III, MD (Children's National Hospital); Michael Morowitz (UPMC Children's Hospital of Pittsburgh); Michael Bell (Children's National Hospital); Randall Burd, MD, PhD (Children's National Hospital); Sabrina Heidemann, MD (Children's Hospital of Michigan); Todd Carpenter, MD (Children's Hospital Colorado).

Availability of data and material. The dataset supporting the conclusions of this article will be made available in the Eunice Kennedy Shriver National Institute of Child Health 
and Human Development Data and Specimen Hub (DASH) repository,

https://dash.nichd.nih.gov/. 


\section{References}

1. Garber N, Watson RS, Linde-Zwirble WT. The size and scope of intensive care for children in the US. Critical Care Medicine 2003;31((Suppl)):A78.

2. Randolph AG, Gonzales CA, Cortellini L, Yeh TS. Growth of pediatric intensive care units in the United States from 1995 to 2001. J Pediatr. 2004;144(6):792-798.

3. Pollack MM, Holubkov R, Funai T, et al. Pediatric intensive care outcomes: development of new morbidities during pediatric critical care. Pediatr Crit Care Med. 2014;15(9):821827.

4. Manning JC, Pinto NP, Rennick JE, Colville G, Curley MAQ. Conceptualizing Post Intensive Care Syndrome in Children-The PICS-p Framework. Pediatr Crit Care Med. 2018;19(4):298-300.

5. Fink EL, Tasker RC. Brain-related outcome measures in trials recruiting critically-ill children. Curr Opin Pediatr. 2019;31(6):775-782.

6. Maddux AB, Pinto N, Fink EL, et al. A Scoping Review: Domains and Instruments Used to Evaluate Morbidities after Pediatric Critical IIIness [abstract]. Pediatric Critical Care Medicine. 2020;(in press).

7. Duffett M, Choong K, Hartling L, Menon K, Thabane L, Cook DJ. Randomized controlled trials in pediatric critical care: a scoping review. Crit Care. 2013;17(5):R256.

8. Taylor A, Butt W, Ciardulli M. The functional outcome and quality of life of children after admission to an intensive care unit. Intensive Care Med. 2003;29(5):795-800.

9. Pinto NP, Rhinesmith EW, Kim TY, Ladner PH, Pollack MM. Long-Term Function After Pediatric Critical Illness: Results From the Survivor Outcomes Study. Pediatr Crit Care Med. 2017;18(3):e122-e130.

10. Duffett M, Choong K, Foster J, et al. High-Quality Randomized Controlled Trials in Pediatric Critical Care: A Survey of Barriers and Facilitators. Pediatr Crit Care Med. 2017;18(5):405-413.

11. Ong C, Lee JH, Leow MK, Puthucheary ZA. Functional Outcomes and Physical Impairments in Pediatric Critical Care Survivors: A Scoping Review. Pediatr Crit Care Med. 2016;17(5):e247-259.

12. Marino BS, Lipkin PH, Newburger JW, et al. Neurodevelopmental outcomes in children with congenital heart disease: evaluation and management: a scientific statement from the American Heart Association. Circulation. 2012;126(9):1143-1172.

13. Merritt C, Menon K, Agus MSD, et al. Beyond Survival: Pediatric Critical Care Interventional Trial Outcome Measure Preferences of Families and Healthcare Professionals. Pediatric critical care medicine : a journal of the Society of Critical Care Medicine and the World Federation of Pediatric Intensive and Critical Care Societies. 2018;19(2):e105-e111.

14. Pasek TA, Burns C, Treble-Barna A, et al. Important Outcomes for Parents of Critically III Children. Critical care nurse. 2019;39(3):74-79.

15. Williamson PR, Altman DG, Blazeby JM, et al. Developing core outcome sets for clinical trials: issues to consider. Trials. 2012;13:132. 
16. Turnbull AE, Sepulveda KA, Dinglas VD, Chessare CM, Bingham CO, 3rd, Needham DM. Core Domains for Clinical Research in Acute Respiratory Failure Survivors: An International Modified Delphi Consensus Study. Crit Care Med. 2017;45(6):1001-1010.

17. Needham DM, Sepulveda KA, Dinglas VD, et al. Core Outcome Measures for Clinical Research in Acute Respiratory Failure Survivors. An International Modified Delphi Consensus Study. American journal of respiratory and critical care medicine. 2017;196(9):1122-1130.

18. Kirkham JJ, Davis K, Altman DG, et al. Core Outcome Set-STAndards for Development: The COS-STAD recommendations. PLoS Med. 2017;14(11):e1002447.

19. Kirkham JJ, Gorst S, Altman DG, et al. COS-STAR: a reporting guideline for studies developing core outcome sets (protocol). Trials. 2015;16:373.

20. Williamson PR, Altman DG, Bagley $\mathrm{H}$, et al. The COMET Handbook: version 1.0. Trials. 2017;18(Suppl 3):280.

21. Fink EL, Jarvis JM, Maddux AB, et al. Development of a Core outcome set for Pediatric critical care outcomes research. Contemp Clin Trials. 2020:105968.

22. Olson L, Zickmund S, Galyean P, et al. PICU Outcomes: A Qualitative Study of Teen and Family Priorities (abstract) Pediatric Critical Care Medicine. 2020;(in press).

23. Diamond IR, Grant RC, Feldman BM, et al. Defining consensus: a systematic review recommends methodologic criteria for reporting of Delphi studies. J Clin Epidemiol. 2014;67(4):401-409.

24. Gershon RC, Rothrock N, Hanrahan R, Bass M, Cella D. The use of PROMIS and assessment center to deliver patient-reported outcome measures in clinical research. $J$ Appl Meas. 2010;11(3):304-314.

25. Guyatt GH, Oxman AD, Vist GE, et al. GRADE: an emerging consensus on rating quality of evidence and strength of recommendations. BMJ. 2008;336(7650):924-926.

26. Levels of neonatal care. Pediatrics. 2012;130(3):587-597.

27. Patient- and family-centered care and the pediatrician's role. Pediatrics. 2012;129(2):394-404.

28. Snyder CF, Jensen RE, Segal JB, Wu AW. Patient-reported outcomes (PROs): putting the patient perspective in patient-centered outcomes research. Med Care. 2013;51(8 Suppl 3):S73-79.

29. Clarke M, Williamson PR. Core outcome sets and systematic reviews. Syst Rev. 2016;5:11.

30. Colville G, Pierce C. Patterns of post-traumatic stress symptoms in families after paediatric intensive care. Intensive Care Med. 2012;38(9):1523-1531.

31. Wiens MO, Pawluk S, Kissoon N, et al. Pediatric post-discharge mortality in resource poor countries: a systematic review. PLoS One. 2013;8(6):e66698.

32. Choi J, Hoffman LA, Schulz R, et al. Self-reported physical symptoms in intensive care unit (ICU) survivors: pilot exploration over four months post-ICU discharge. J Pain Symptom Manage. 2014;47(2):257-270.

33. Watson RS, Asaro LA, Hutchins L, et al. Risk Factors for Functional Decline and Impaired Quality of Life after Pediatric Respiratory Failure. Am J Respir Crit Care Med. 2019;200(7):900-909. 
34. Kachmar AG, Irving SY, Connolly CA, Curley MAQ. A Systematic Review of Risk Factors Associated With Cognitive Impairment After Pediatric Critical IIIness. Pediatr Crit Care Med. 2018;19(3):e164-e171.

35. Meert K, Slomine BS, Christensen JR, et al. Burden of caregiving after a child's in-hospital cardiac arrest. Resuscitation. 2018;127:44-50.

36. Anderson VA, Catroppa C, Dudgeon P, Morse SA, Haritou F, Rosenfeld JV. Understanding predictors of functional recovery and outcome 30 months following early childhood head injury. Neuropsychology. 2006;20(1):42-57.

37. Williams CN, Hartman ME, Guilliams KP, et al. Postintensive Care Syndrome in Pediatric Critical Care Survivors: Therapeutic Options to Improve Outcomes After Acquired Brain Injury. Curr Treat Options Neurol. 2019;21(10):49.

38. Williams CN, Hartman ME, McEvoy CT, et al. Sleep-Wake Disturbances After Acquired Brain Injury in Children Surviving Critical Care. Pediatr Neurol. 2019.

39. Prinsen CA, Vohra S, Rose MR, et al. Core Outcome Measures in Effectiveness Trials (COMET) initiative: protocol for an international Delphi study to achieve consensus on how to select outcome measurement instruments for outcomes included in a 'core outcome set'. Trials. 2014;15:247. 


\section{Figure Legends}

Figure 1. PICU Core Outcome Set: Modified Delphi Survey Flow Diagram

Figures $2 \underline{a}$ and $2 b$. Differences in scores from Round 1 to round 2 by PICU Core Outcome Set stakeholder group.

Supplemental Figures $1 \mathrm{a}$ and $1 \mathrm{~b}$. Delphi Survey Round 2 results by stakeholder group. Results presented as median (interquartile range) with mean identified by open diamonds and outliers identified by open circles. 